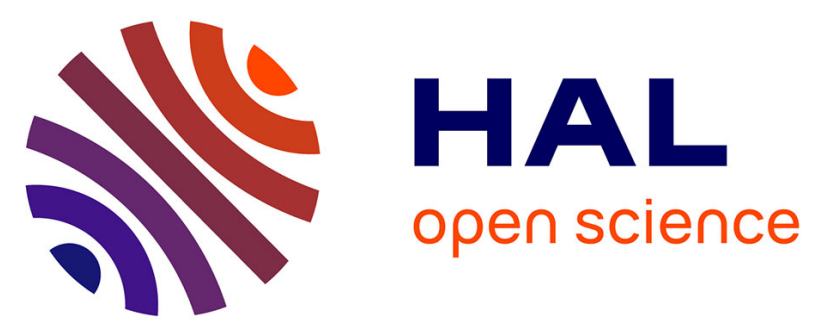

\title{
En reconstruction mammaire : intérêt du dépistage du portage de Staphylococcus aureus dans la prévention de l'infection du site opératoire
}

\author{
Sophie Rouquette, F. Cherrière, A. Vagneur, F. Godey, S. Sun, Anne \\ Jolivet-Gougeon, H. Mesbah, Odile Audrain, C Bendavid
}

\section{To cite this version:}

Sophie Rouquette, F. Cherrière, A. Vagneur, F. Godey, S. Sun, et al.. En reconstruction mammaire : intérêt du dépistage du portage de Staphylococcus aureus dans la prévention de l'infection du site opératoire. Annales de Chirurgie Plastique Esthétique, 2015, 60 (6), pp.490-494. 10.1016/j.anplas.2015.07.007 . hal-01187136

\section{HAL Id: hal-01187136 \\ https://hal-univ-rennes1.archives-ouvertes.fr/hal-01187136}

Submitted on 26 Nov 2015

HAL is a multi-disciplinary open access archive for the deposit and dissemination of scientific research documents, whether they are published or not. The documents may come from teaching and research institutions in France or abroad, or from public or private research centers.
L'archive ouverte pluridisciplinaire HAL, est destinée au dépôt et à la diffusion de documents scientifiques de niveau recherche, publiés ou non, émanant des établissements d'enseignement et de recherche français ou étrangers, des laboratoires publics ou privés. 


\section{En reconstruction mammaire : intérêt du dépistage du portage de Staphylococcus}

\section{aureus dans la prévention de l’infection du site opératoire.}

Screening for carriage of Staphylococcus aureus prior to mammary reconstruction in the prevention of surgical site infection

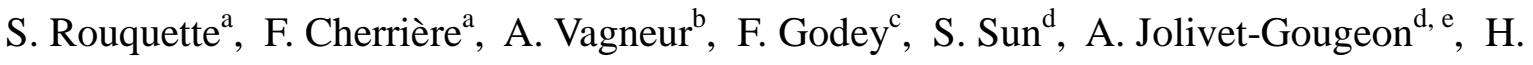

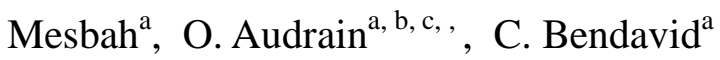

a Département de chirurgie, CLCC centre Eugène-Marquis, CS44229, 35042 Rennes

cedex, France

${ }^{\mathrm{b}}$ Département hygiène et qualité, CLCC centre Eugène-Marquis, CS44229, 35042 Rennes cedex, France

c Département de biologie, CLCC centre Eugène-Marquis, CS44229, 35042 Rennes cedex, France

${ }^{\mathrm{d}}$ Pôle biologie CHU Pontchaillou, 35000 Rennes, France

${ }^{\text {e }}$ EA 1254 microbiologie, université de Rennes 1, 35043 Rennes, France

\section{Résumé}

L'incidence des infections sur prothèse après reconstruction mammaire est de l'ordre de $4 \%$ à $13 \%$ selon la littérature. Chez les patients opérés, le staphylocoque doré (S. aureus) est l'espèce bactérienne la plus souvent responsable d'infections du site opératoire. En chirurgie cardiaque, le dépistage du portage de $S$. aureus et la décontamination préopératoire sont pratiqués en routine avant chirurgie prothétique. Une étude prospective, au CLCC à Rennes a été réalisée entre janvier 2011 et décembre 2013. En reprenant nos séries, nous avons remarqué que les taux d'infections sur prothèse étaient de l'ordre de 5,92 \% en 2008 avec un taux d'ISO à $S$. aureus de 3,61\%. Le dépistage systématique pour les reconstructions par prothèse a été réalisé pour évaluer l'impact d'une décontamination préopératoire chez les patientes porteuses de $S$. aureus. Ce dépistage a été fait chez 381 patientes : 17,8\% des patientes étaient porteuses de $S$. aureus ; 11 patientes ont fait une ISO (soit un taux d'incidence de $2,88 \%$ ) ; 5 patientes ont fait une ISO à $S$. aureus (soit une incidence d'ISO à $S$. aureus de $1,3 \%$ ). La mise en place de la procédure de dépistage a permis une baisse du taux d' ISO de $5,92 \%$ à $1,46 \%$ avec un passage du taux d' ISO à S. aureus de 3,60\% à 0,72\%. Ces résultats sont encourageants et d'autres études seront nécessaires pour démontrer l'efficacité d'une décontamination préopératoire chez les patientes porteuses de $S$. aureus avant pose de prothèse. 


\section{Summary}

The incidence of prosthesis infections after breast reconstruction is of the order of $4 \%$ to $13 \%$ according to the literature. In surgical patients, Staphylococcus aureus (S. aureus) is the bacterial species most often responsible for surgical site infections. In cardiac surgery, screening for carriage of $S$. aureus and preoperative decontamination are carried out routinely before prosthetic surgery. We retrospectively reviewed data from patients at our institution between January 2011 and December 2013. Our series showed that the prosthesis infection rates were in the range of $5.92 \%$ in 2008 with an ISO rate of $S$. aureus $3.61 \%$. Routine screening for prosthetic reconstructions was performed to assess the impact of preoperative decontamination patients in carriers of $S$. aureus. This screening was done in 381 patients: $17.8 \%$ of patients were carriers of $S$. aureus ; 11 patients have an ISO (or an incidence rate of $2.88 \%$ ) ; 5 patients have an ISO S. aureus (an incidence of S. aureus ISO $1.3 \%$ ). The introduction of the screening process, allowed a drop of $5.92 \%$ ISO rate at $1.46 \%$ with a passage of $S$. aureus SSI rates of $3,60 \%$ to $0.72 \%$. In the near future, studies are needed to confirm these encouraging results, to demonstrate the efficacy of preoperative decontamination in carriers of $S$. aureus patients before laying prosthesis.

\section{Introduction}

L'infection du site opératoire (ISO) est la complication la plus fréquente des interventions chirurgicales [1]. Elle représente la troisième cause des infections nosocomiales [2]. Sa surveillance est assurée en France par les CCLIN (Centre de Coordination de la Lutte contre les Infections Nosocomiales). Le staphylocoque doré (S.aureus) est le pathogène le plus fréquemment responsable d'ISO, surtout en chirurgie propre [3]. L'utilisation de matériel prothétique augmente le risque d'infection post-opératoire de trois à six. En chirurgie reconstructrice mammaire, avec utilisation d'implant prothétique, l'incidence des infections est de l'ordre de quatre à treize \% selon la littérature [4].

Comme le met en évidence le rapport ISO du CCLIN Ouest 2013, S. aureus $(\mathrm{n}=302)$ est la principale espèce bactérienne responsable d'ISO (22,7 \% des cas), suivi d'E.coli (17 \%), E.faecalis (7.3\%), S.epidermidis (6.4\%), P.aeruginosa (5,4\%). La littérature est en accord avec les données du CCLIN Ouest: S.aureus est la principale espèce bactérienne retrouvée, notamment en chirurgie cardiaque [5] et en chirurgie orthopédique [6]. 
En chirurgie mammaire, S.aureus est la première espèce bactérienne responsable d'ISO, selon plusieurs études [7,8], avec un taux plus élevé de Staphylococcus aureus métisensible (SAMS) par rapport au Staphylococcus aureus résistant à la méticilline (SARM).

Le site de portage préférentiel de $S$. aureus se situe au niveau des fosses nasales antérieures (au niveau du vestibulum nasi) [9]. Sa présence multiplie le risque de trois à six [10,11]. Dans la population générale il est intermittent dans $30 \%$ des cas, absent dans $50 \%$ et permanent dans $20 \%$. Dans ce dernier cas, il est associé à une charge bactérienne élevée et un risque plus important d'infection à S. aureus [9]. Il existe une relation étroite entre portage cutané et portage nasal : le portage cutané au niveau des mains passe de $27 \%$ dans la population générale à 90\% chez les porteurs nasaux permanents de S.aureus [12].

L'impact du dépistage nasal et de la décontamination du portage à S.aureus sur la diminution des ISO a fait l'objet de nombreuses études dont Lepelletier et al. (2013) ont fait la synthèse [13]. Seule une étude récente de Bode et al. [14] a démontré l'efficacité de la décontamination nasale par mupirocine chez les porteurs de S.aureus, montrant aussi l'intérêt du dépistage rapide par PCR à l'admission (réduction de 58\% du taux d'infection à S.aureus, OR 0,42, IC95\% 0,23-0,75).

Afin de mettre en évidence l'intérêt de la décolonisation du S. aureus avant la chirurgie, la Société Française d'Hygiène Hospitalière (SFHH) a actualisé en octobre 2013 ses recommandations de bonne pratique sur la gestion préopératoire du risque infectieux, près de 10 ans après la conférence de consensus de 2004. Deux thèmes principaux ont été révisés : (1) la préparation cutanée (douche préopératoire, dépilation, détersion et antisepsie du site opératoire, utilisation des champs opératoires imprégnés d'antiseptiques) et (2) le dépistage et la décolonisation nasale des patients porteurs de S.aureus. Selon la méthode Grade [15], la décolonisation n'est recommandée que chez les patients bénéficiant d'une chirurgie cardiaque. Aucune recommandation n'a été émise pour les autres types de 
chirurgie par manque de niveau de preuve des différentes études. Ces recommandations ont été basées sur une revue rigoureuse de la littérature scientifique [16].

En reprenant les données de nos séries de 2008-2009, nous avons remarqué que nos taux d'infections sur prothèse étaient de l'ordre de $4,93 \%$ mais avec un taux d'ISO à $S$. aureus de 3,61 (304 patientes opérées, 15 ISO dont 11 ISO à S. aureus). Le moyen de diminuer les infections post-opératoires était donc de diminuer le risque d'infections à $S$. aureus. L'objectif de ce travail est donc d'étudier l'incidence des ISO en chirurgie de reconstruction mammaire, chez les porteurs de $S$. aureus ayant eu une éradication du portage avant la chirurgie.

\section{Matériels et méthodes}

Notre étude prospective a été réalisée de janvier 2011 à décembre 2013 au Centre de Lutte Contre le Cancer (CLCC) Eugène Marquis de Rennes. Toutes les patientes bénéficiant d'une reconstruction par prothèse ou expandeur ont été dépistées. Il pouvait s'agir d'une reconstruction immédiate ou différée dans le cadre d'une prise en charge oncologique ou préventive. L'ensemble des interventions a été réalisé par deux chirurgiens aux pratiques semblables. Un consentement oral, après remise d'une fiche informative, a

été recueilli. Le dépistage a été réalisé lors de la consultation préopératoire par un écouvillonnage nasal. Toutes les patientes porteuses du $S$. aureus ont bénéficié d'une décontamination : douche quotidienne à la Chlorhexidine les cinq jours qui précédaient l'intervention chirurgicale et application nasale de mupirocine trois fois par jour. La 
douche préopératoire, la décontamination opératoire et les soins de drains de Redon postopératoires ont également été fait à la Chlorhexidine.

La recherche de $S$. aureus a été réalisée au laboratoire de Bactériologie par isolement sur milieu sélectif de Chapman (Oxoid) et identification par spectrométrie de masse (MALDI TOF, Biotyper®, Bruker). La sensibilité à la mupirocine a été testée selon les recommandations du CA-SFM EUCAST [17] (disques Biorad).

L'incidence des ISO a été retrouvée grâce aux déclarations faites dans le service. Étaient considérées comme ISO les infections survenant dans l'année suivant la mise en place de l'implant associé à au moins un des critères suivant: présence de pus, germes mis en évidence, abcès ou signes infectieux (hyperthermie, rougeur, chaleur...), diagnostique d'infection porté par le chirurgien.

Les résultats obtenus ont ensuite été comparés à une cohorte rétrospective de patientes aux mêmes critères d'inclusion, opérées par les deux mêmes chirurgiens mais n'ayant pas bénéficié de dépistage (année 2008, 2009 et 2010).

Les statistiques ont été réalisées grâce au Test du Chi2 normal et au test exact de Fisher.

\section{Résultats}

Entre 2011 et 2013, 381 patientes ont bénéficié d'une chirurgie de reconstruction mammaire dont $17,8 \%$ (68) étaient porteuses du S. Aureus.

Avec la mise en place de la procédure de dépistage (janvier 2011), notre taux d'ISO pour les reconstructions avec prothèse est passé de 5,92\% en 2008 à 1,46 \% en 2013 (Figure 1A) avec un passage du taux d'ISO à S. aureus de 3,6 \% en 2008 à 0,72\% en 2013 (Figure 1B). Concernant le nombre d'ISO entre 2011 et 2013, 11 patientes ont présenté une ISO (5 en 2011, 4 en 2012, 2 en 2013) et les bactéries ont été identifiées dans 10 cas sur $11: S$. 
aureus : trois patientes à dépistage nasal positif et décontamination à la mupirocine réalisée et deux patientes à dépistage nasal négatif (Tableau 1).

Tableau 1 : Détails des ISO de 2011 à 2013

\begin{tabular}{|l|l|l|l|}
\hline & dépistage & décontamination & Germe retrouvé \\
\hline 2011 & positif & oui & Klebsiella Oxtoca \\
\hline 2011 & négatif & non & S.aureus \\
\hline 2011 & positif & oui & S.aureus \\
\hline 2011 & négatif & non & Enterobacter Cloacae \\
\hline 2012 & positif & oui & S.aureus \\
\hline 2012 & négatif & non & Non identifié \\
\hline 2012 & négatif & non & S.aureus \\
\hline 2012 & négatif & non & Streptocoque \\
\hline 2013 & négatif & non & Streptocoque \\
\hline & non & Serratia Marecescens \\
\hline
\end{tabular}

Les résultats ont été suivis pendant l'année 2014 et confirment la diminution des infections à $S$. aureus.

\section{Discussion}

Le portage nasal de Staphylococcus aureus est un facteur de risque d'ISO démontré notamment en chirurgie cardiaque [18]. La réduction des ISO après décolonisation des 
porteurs est démontrée dans cette spécialité [14]. En chirurgie générale il est simplement démontré une réduction du nombre d'infection nosocomiale [19]. La décolonisation corporelle et oropharyngée par un produit antiseptique efficace contre $S$. Aureus est une des mesures de prévention de l'ISO parmi d'autres. Quatre essais randomisés, Garcia et al. [20], Segers et al. [21], Konvalinka et al. [22], Bode et al. [14], et trois études observationnelles, Cimochovski et al [23], Nicholson et al. [24], Jog et al. [25], ont étudié l'intérêt d'une décolonisation préopératoire chez les porteurs de $S$. aureus avant chirurgie cardiaque. Sur ces trois études, deux présentaient des effets positifs : Cimochovski et al. en 2001 [23] avec un RR de 0,34 (IC=0,16-0,75) et Nicholson en 2006 [24] avec un RR de 0,22 (IC=0,07-0,66). La $3^{\text {ème }}$ étude, Jog et al. [25,26] ne montrait pas d'effet positif significatif, avec un $\mathrm{RR}$ de 0,34 (IC=0,09-1,28). La méta-analyse incluant ces trois études observationnelles montrait un bénéfice de la décolonisation nasale par mupirocine avec un $\mathrm{RR}$ de 0,30 (IC=0,17-0,54). Au total, les résultats de la méta-analyse regroupant l'ensemble des sept études étaient significativement positifs avec un $\mathrm{RR}=0,44$ ( $\mathrm{IC}=0,26$ 0,74). Il est donc recommandé de réaliser une décolonisation du portage de $S$. aureus chez les patients bénéficiant d'une chirurgie cardiaque pour réduire le taux d'infection du site opératoire à S. aureus.

Dans la littérature, aucune étude n'a évalué l'intérêt du dépistage et de la décolonisation de S. aureus avant une reconstruction mammaire par pose de prothèse. Cependant, Craft et al. (2012) [26] ont proposé un protocole visant à réduire les infections après reconstruction mammaire, en se basant sur les données de la littérature. D'autre part, l'ABS (Association of Breast Surgery) qui a publié récemment en 2012 les Guidelines for Best Practice, préconise, chez les patients avec un dépistage nasal positif à S.aureus, une décolonisation nasale par la mupirocine et corporelle par la chlorhexidine [27]. 
Parmi les trois patientes ayant développé une ISO à S. Aureus, alors que la décolonisation avait été faite, les causes possibles de l'échec de la décontamination peuvent être une administration incomplète de mupirocine ou une insuffisance de la décontamination corporelle voire oropharyngée, comme l'évoquent Lepelletier et al. dans leur revue [13].

On constate une première baisse importante des ISO en 2010 dans notre centre avant la mise en place du dépistage nasale. Cela pourrait s'expliquer par la mise en place d'une nouvelle procédure pour les ponctions de lymphocèle.

Le portage de S. aureus peut-être aussi extra nasal : on dénombre $20 \%$ de porteurs digestifs et $12 \%$ de porteurs oropharyngés sans portage nasal [28]. Se pose alors la question suivante : est-il nécessaire de dépister différents sites anatomiques de portage?

Concernant la possibilité de résistance acquise à la mupirocine, deux études (Perl et al.[19] et Fawley et al. [29]) n'ont pas réussi à prouver une augmentation de la résistance sur des souches isolées sur une période de quatre ans.

On retrouve, ces dernières années, différentes études montrant l'intérêt d'une reprise chirurgicale en urgence et en un temps lorsqu'une infection est constatée. Il est effectué un prélèvement bactériologique puis un lavage et un changement d'implant, couplé à une antibiothérapie adaptée [30,31].

Devant la baisse des ISO des 3 dernières années, le dépistage du portage de toutes les patientes bénéficiant d'une reconstruction est poursuivie dans notre centre. Ces résultats satisfaisants ont été présentés dans différents congrès et ont incité d'autres centres à dépister leurs patientes. De nombreux autres facteurs de risque d'ISO existent : âge, IMC, diabète, tabagisme, durée d'intervention et d'hospitalisation, absence d'antibioprophylaxie... Une étude intégrant l'ensemble de ces facteurs de risque associés au dépistage et au traitement ou non du portage de $S$ aureus est en cours dans différents centres français. 


\section{Conclusion}

Une baisse du taux d'ISO a donc été constatée depuis la mise en place du dépistage du portage nasale du S.Aureus et de sa décontamination.

Ces résultats encourageants nous incitent à poursuivre cette démarche avant reconstruction mammaire avec pose de prothèse. D'autres études prospectives multicentriques, randomisées seront nécessaires afin de démontrer l'efficacité d'une décontamination préopératoire chez les patientes porteuses du $S$. aureus avant pose de prothèse. Une étude multicentrique regroupant six Centres de Lutte Contre le Cancer français est actuellement en cours afin d'évaluer les résultats à plus grande échelle de la décontamination préopératoire chez les patientes porteuses du S.aureus.

Aucun conflit d'intérêt 
Légendes des figures :

\section{Figure 1}

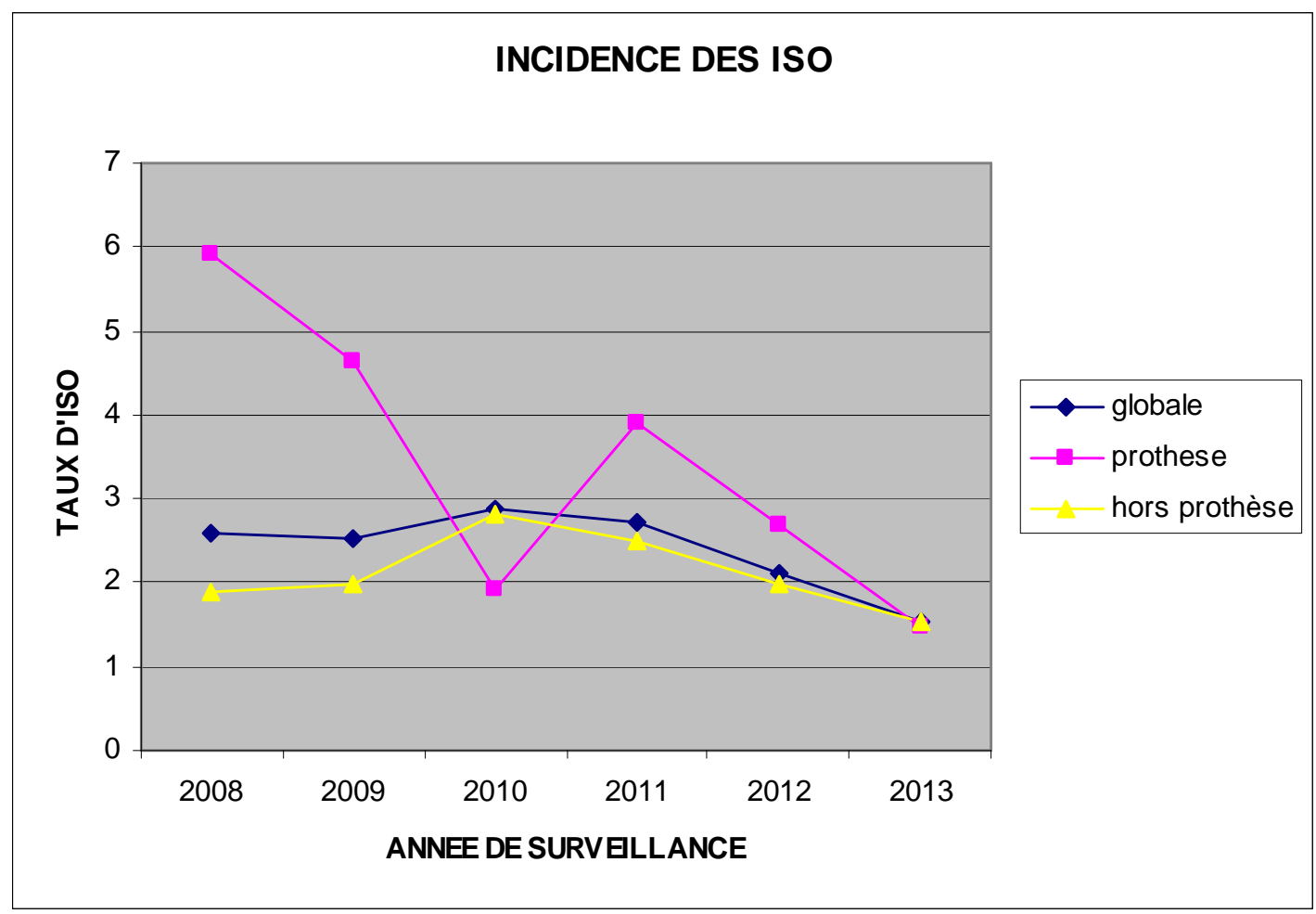

Figure 1 : Evolution de l'incidence globale des ISO entre 2008 et 2013

\section{Figure 2}




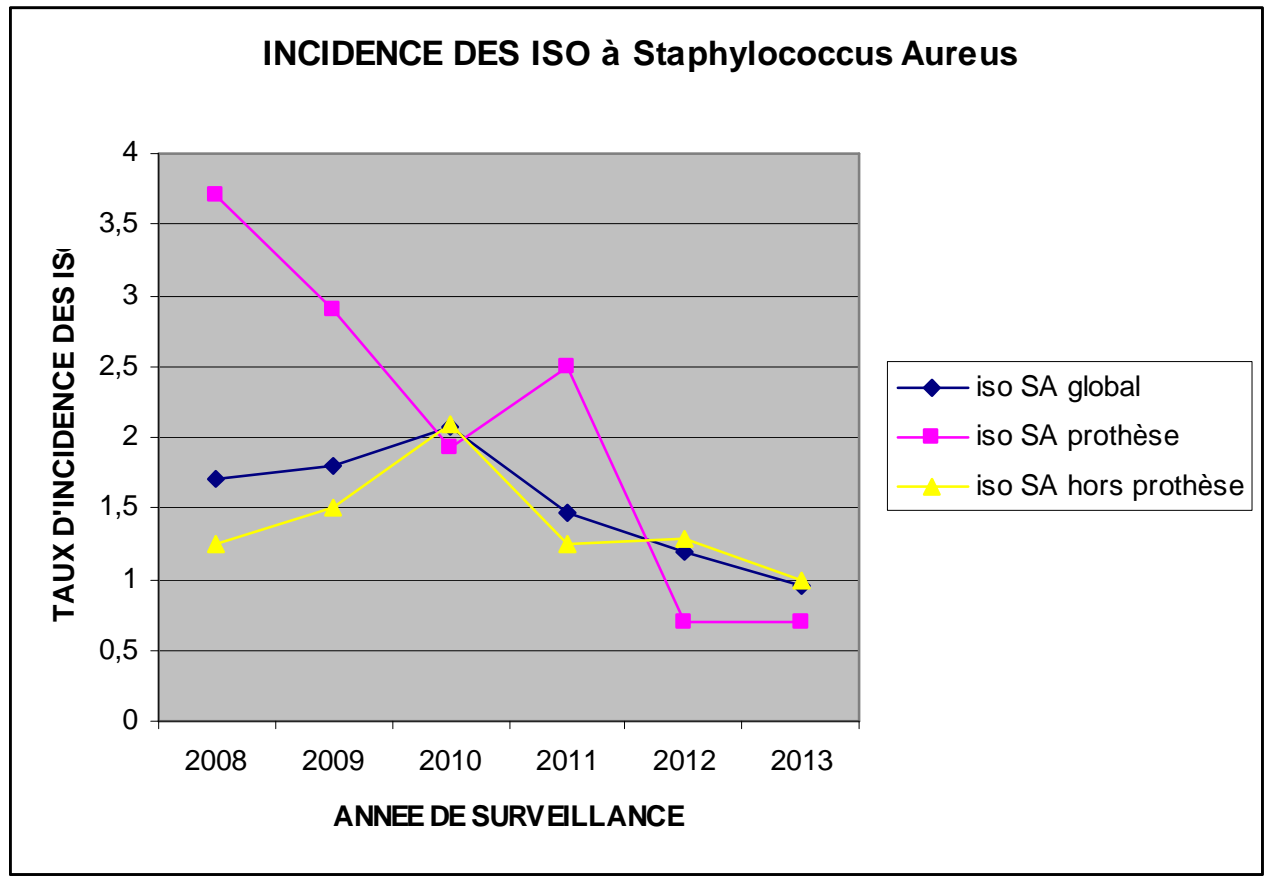

Figure 2 : Evolution de l'incidence globale des ISO à S. aureus entre 2008 et 2013 


\section{Réferences}

[1] C-CLIN Est. Réseau ISO-Raisin : Surveillance des Infections du site opératoire. 126. 2013. Vandoeuvre-lès-Nancy, C-CLIN-Est.

[2] Thiolet JM, Lacavé L, Jarno P et al. Prévalence des infections nosocomiales, France, 2006. B E H 2007;51-52:5294-431.

[3] Aupée M, Tanguy J, Garreau N, Dixon MP. Surveillance Agrégée des Infections du Site Opératoire. Résultats interrégion Ouest Année 2014. 1-22. 2015. C CLINOuest.

[4] Nahabedian MY, Tsangaris T, Momen B, Manson PN. Infectious complications following breast reconstruction with expanders and implants. Plast Reconstr Surg 2003;112(2):467-476.

[5] Lepelletier D, Bourigault C, Roussel JC et al. Epidemiology and prevention of surgical site infections after cardiac surgery. Med Mal Infect 2013;43(10):403-409.

[6] Kalmeijer MD, van Nieuwland-Bollen E, Bogaers-Hofman D, de Baere GA. Nasal carriage of Staphylococcus aureus is a major risk factor for surgical-site infections in orthopedic surgery. Infect Control Hosp Epidemiol 2000;21(5):319-323.

[7] Mukhtar RA, Throckmorton AD, Alvarado MD et al. Bacteriologic features of surgical site infections following breast surgery. Am J Surg 2009;198(4):529-531.

[8] Viola GM, Raad II, Rolston KV. Breast tissue expander-related infections: perioperative antimicrobial regimens. Infect Control Hosp Epidemiol 2014;35(1):75-81. 
[9] Kluytmans J, van BA, Verbrugh H. Nasal carriage of Staphylococcus aureus: epidemiology, underlying mechanisms, and associated risks. Clin Microbiol Rev 1997;10(3):505-520.

[10] Kluytmans JA, Mouton JW, Ijzerman EP et al. Nasal carriage of Staphylococcus aureus as a major risk factor for wound infections after cardiac surgery. $\mathrm{J}$ Infect Dis $1995 ; 171(1): 216-219$

[11] Société Française d'Hygiène Hospitalière. Gestion préoperatoire du risque infectieux. Conference de consensus. Hygienes , 1-216. 2004. SFHH.

[12] Wertheim HF, Melles DC, Vos MC et al. The role of nasal carriage in Staphylococcus aureus infections. Lancet Infect Dis 2005;5(12):751-762.

[13] Lepelletier D, Lucet JC. Impact du dépistage et de la décontamination sur la prévention des infections du site opératoire à Staphylococcus aureus. Journal des anti-infectieux 2011;13(4):209-2016.

[14] Bode LG, Kluytmans JA, Wertheim HF et al. Preventing surgical-site infections in nasal carriers of Staphylococcus aureus. N Engl J Med 2010;362(1):9-17.

[15] Guyatt GH, Oxman AD, Vist GE et al. GRADE: an emerging consensus on rating quality of evidence and strength of recommendations. BMJ 2008;336(7650):924926.

[16] Société Française d'Hygiène Hospitalière. Mise à jour de la conférence de consensus. Gestion préopératoire du risque infectieux, 2013. Hygiènes 2013;21(4):1-112.

[17] CA-SFM. Comité de l'Antibiogramme de la Société Française de Microbiologie. Recommandations 2014. 2014. 
[18] Verhoeven PO, Gagnaire J, Botelho-Nevers E et al. Detection and clinical relevance of Staphylococcus aureus nasal carriage: an update. Expert Rev Anti Infect Ther 2014;12(1):75-89.

[19] Perl TM, Cullen JJ, Wenzel RP et al. Intranasal mupirocin to prevent postoperative Staphylococcus aureus infections. N Engl J Med 2002;346(24):1871-1877.

[20] Garcia AM, Villa MV, Escudero ME et al. [Use of nasal mupirocin for Staphylococcus aureus: effect on nasal carriers and nosocomial infections]. Biomedica 2003;23(2):173-179.

[21] Segers P, Speekenbrink RG, Ubbink DT, van Ogtrop ML, de Mol BA. [Prevention of nosocomial infections after cardiac surgery by decontamination of the nasopharynx and oropharynx with chlorhexidine; a prospective, randomised study]. Ned Tijdschr Geneeskd 2008;152(13):760-767.

[22] Konvalinka A, Errett L, Fong IW. Impact of treating Staphylococcus aureus nasal carriers on wound infections in cardiac surgery. J Hosp Infect 2006;64(2):162-168.

[23] Cimochowski GE, Harostock MD, Brown R, Bernardi M, Alonzo N, Coyle K. Intranasal mupirocin reduces sternal wound infection after open heart surgery in diabetics and nondiabetics. Ann Thorac Surg 2001;71(5):1572-1578.

[24] Nicholson MR, Huesman LA. Controlling the usage of intranasal mupirocin does impact the rate of Staphylococcus aureus deep sternal wound infections in cardiac surgery patients. Am J Infect Control 2006;34(1):44-48.

[25] Jog S, Cunningham R, Cooper S et al. Impact of preoperative screening for meticillin-resistant Staphylococcus aureus by real-time polymerase chain reaction in patients undergoing cardiac surgery. J Hosp Infect 2008;69(2):124-130. 
[26] Craft RO, Damjanovic B, Colwell AS. Evidence-based protocol for infection control in immediate implant-based breast reconstruction. Ann Plast Surg 2012;69(4):446-450.

[27] Rainsburt D, Winnett A. Oncoplastic Breast Reconstruction : Guidelines for Best Practice. London: ABS,BAPRAS; 2012.

[28] Acton DS, Plat-Sinnige MJ, van WW, de GN, van BA. Intestinal carriage of Staphylococcus aureus: how does its frequency compare with that of nasal carriage and what is its clinical impact? Eur J Clin Microbiol Infect Dis 2009;28(2):115127.

[29] Fawley WN, Parnell P, Hall J, Wilcox MH. Surveillance for mupirocin resistance following introduction of routine peri-operative prophylaxis with nasal mupirocin. $\mathbf{J}$ Hosp Infect 2006;62(3):327-332.

[30] Missana MC, Blot F, Germain M. Complication infectieuse sur prothèse en chirurgie reconstructrice mammaire : quelle attitude thérapeutique adopter ? Ann Chir Plast Esthet 2012;57(1):16-24.

[31] Quilichini J, Guihard T, Le MP. Sauvetage de prothèses en reconstruction mammaire : à propos de sept cas consécutifs. Ann Chir Plast Esthet 2010;55(6):578-582. 